Portland State University

PDXScholar

Civil and Environmental Engineering Master's

Project Reports

Summer 2018

\title{
Lateral Deformation Behavior of Mass Timber Beam to Column Gravity Connection
}

Tyler James Williams

Portland State University

Follow this and additional works at: https://pdxscholar.library.pdx.edu/cengin_gradprojects

Part of the Civil Engineering Commons

Let us know how access to this document benefits you.

\section{Recommended Citation}

Williams, Tyler James, "Lateral Deformation Behavior of Mass Timber Beam to Column Gravity Connection" (2018). Civil and Environmental Engineering Master's Project Reports. 44.

https://doi.org/10.15760/CCEMP.43

This Project is brought to you for free and open access. It has been accepted for inclusion in Civil and Environmental Engineering Master's Project Reports by an authorized administrator of PDXScholar. Please contact us if we can make this document more accessible: pdxscholar@pdx.edu. 
LATERAL DEFORMATION BEHAVIOR OF MASS TIMBER BEAM TO COLUMN GRAVITY CONNECTION

BY

TYLER JAMES WILLIAMS

A research project report submitted in partial fulfillment of the requirement for the degree of

\section{MASTER OF SCIENCE \\ IN}

CIVIL AND ENVIRONMENTAL ENGINEERING

Project Advisor:

Peter Dusicka, Ph.D., PE

Portland State University

(C)2018 


\section{ACKNOWLEDGEMENTS}

The authors wish to acknowledge the National Science Foundation, Award \#1563612; Eric McDonnel, PE, SE and Reid Zimmerman, PE, SE from KPFF Consulting Engineers in Portland, Oregon; Lever Architecture, StructureCraft; and the Framework development team at project $^{\wedge}$ for their contributions and support of this research. 


\begin{abstract}
A beam-to-column connection consisting of glulam beam and column and cross laminated timber floor slab was developed and experimentally evaluated for use in buildings that are expected to undergo earthquake-induced lateral drift. The connection utilized a passive gap closure mechanism designed to allow beam rotations during lateral drift while minimizing residual separation between the beam and the column following an earthquake. Full scale cyclic experiments were used to validate the connection's minimal rotational stiffness and demonstrate low damage performance for lateral drifts exceeding $4.5 \%$ drift. The connection exhibited no degradation in gravity load capacity throughout the imposed lateral deformations. An analytical model was developed and presented to describe the behavior of the connection including the gap closure mechanism. The experimental data showed that the developed beam to column gravity connection can be a viable seismically resilient solution for mass timber buildings located in medium to high seismic hazard.
\end{abstract}




\section{TABLE OF CONTENTS}

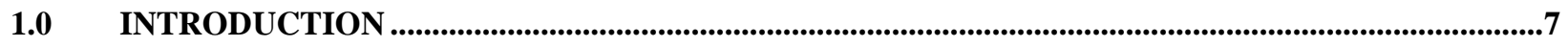

2.0 CONNECTION DESCRIPTION AND TESTING PROCEDURE.......................................................

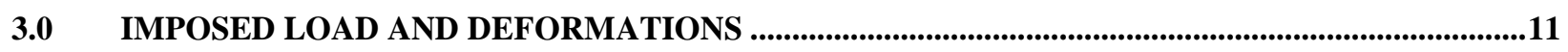

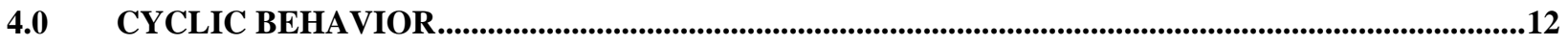

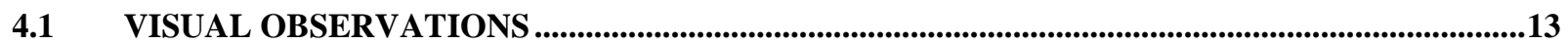

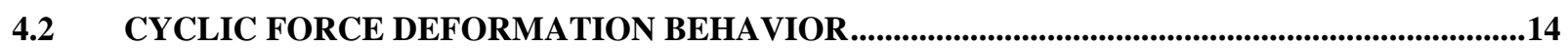

5.0 MODELING OF GAP CLOSURE MECHANISM FORCE-DEFORMATION BEHAVIOR ...............16

6.0 EFFECTIVENESS OF GAP-CLOSURE MECHANISM ................................................................19

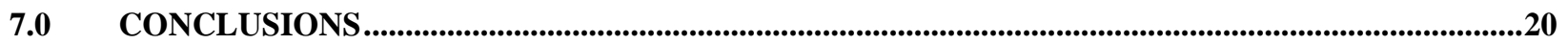

8.0 APPENDIX 1 - BEAM GROWTH EVALUATION ....................................................................................21

9.0 APPENDIX 2 - FURTHER STUDY OF GAP CLOSURE MECHANISM ...............................................23

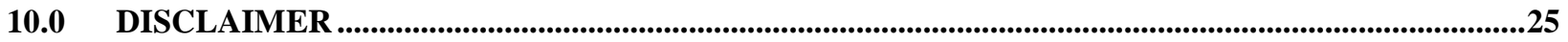

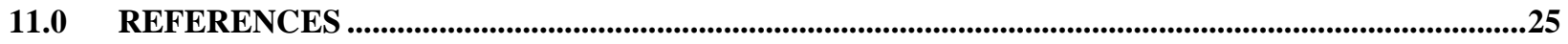

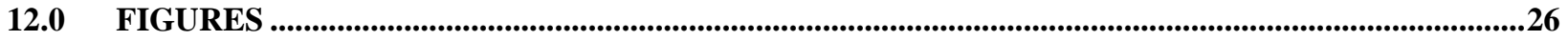

13.0 TABLES .................................................................................................................................................36 


\section{LIST OF TABLES}

TABLE 1 - VARIABLE CONDITIONS FOR EACH BCT CONNECTION TEST. 


\section{LIST OF FIGURES}

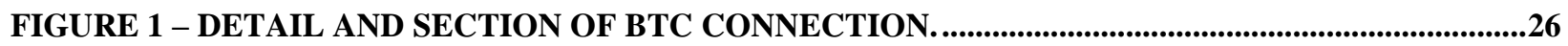

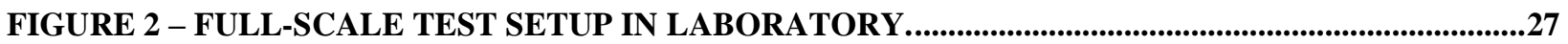

FIGURE 3 - TEST SETUP OVERVIEW AND FREE BODY DIAGRAM. ..........................................................28

FIGURE 4 - DISPLACEMENT HISTORY PROTOCOL MODIFIED FROM CUREE W-02. EXPECTED

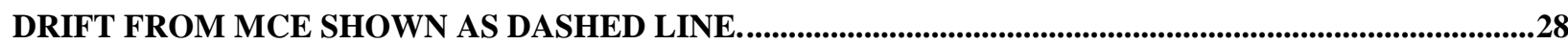

FIGURE 5 -BTC CONNECTION DURING 6\% POSITIVE DRIFT AND VISIBLE INDENTATION IN

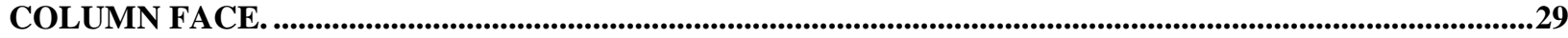

FIGURE 6 - FORCE VS. DIRFT PLOTS FOR BTC CONNECTION TESTS. .....................................................30

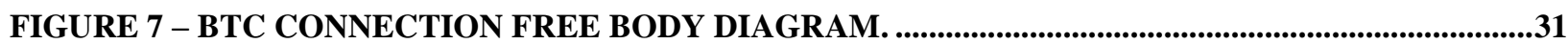

FIGURE 8 - EXPERIMENTAL AND ANALYTICAL CONNECTION FORCE-DISPLACEMENT PLOTS

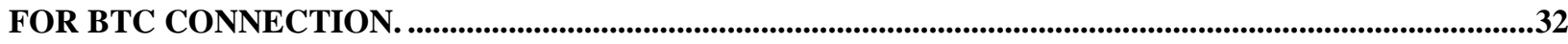

FIGURE 9 - NON-LINEAR DEVELPMENT OF S-SHAPED FORCE-DISPLACEMENT CURVE................33

FIGURE 10 - PEAK AND RESIDUAL GAPS BETWEEN BEAM AND COLUMN. ..........................................34

FIGURE 11 - EXPERIMENTAL AND ANALYTICAL BEAM GROWTH EVALUATION. .............................35

FIGURE 12 - PEAK AND RESIDUAL GAPS BETWEEN BEAM AND COLUMN FOR PARAMETRIC

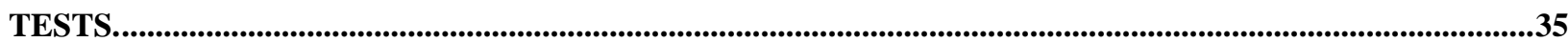




\subsection{INTRODUCTION}

Recent engineering, manufacturing and technological advancements have made mass timber a viable construction material for large and tall buildings in the United States. The appetite for sustainable structural systems, such as mass timber frames and cross laminated timber shear walls, poses new challenges for researchers and engineers seeking to design seismically-resilient structures. A variety of mass timber lateral force resisting systems (LFRS) are currently under development to meet performancebased design criteria. Many such systems place specific emphasis on mitigating damage and accommodating a rapid return to occupancy following design-level seismic events. Rocking, posttensioned cross laminated timber (CLT) shear walls are currently being considered as one possible mass timber LFRS when combined with a glulaminated timber (glulam) beam and column gravity system (Zimmerman and McDonnel, "Framework - A tall re-centering mass timber building in the United States," presented at NZSEE Conference, 2017). However, such rocking systems require special detailing at beam-to-column (BTC) gravity connections to accommodate inter-story drifts without excessive damage to the glulam members at the connection. This paper summarizes the design and experimental evaluation of a BTC connection that was specifically designed to accommodate large lateral drifts with minimal damage, negligible residual displacement and without reduction in gravity load capacity.

While wood is one of the oldest and most frequently-used building materials in many parts of the world, its anisotropic properties pose material-based challenges to constructing tall structures that are economical and capable of withstanding lateral loads in moderate to high-seismic regions. Wood's material structure proves particularly challenging when designing ductile connections between members. Wood is well known to be brittle in both tension perpendicular to the grain and longitudinal shear. A wide range of gravity connections have been developed to accommodate these mechanical limitations, however only recently have engineers and researchers began fully considering the effects of significant seismic lateral drifts that can be imposed on these timber gravity connections. Models have been developed to predict the rotational stiffness of traditional Nuki (mortise) joints used widely throughout Japan and China for 
hundreds of years (Chang, Hsu and Komatsu 2004). Contemporary BTC connections that use dowel-type fasteners and steel plates have been evaluated to determine failure modes and ductility capacities based on a variety of factors (Jorissen and Fragiacomo, 2011). Research has revealed that traditional bolted knife plate BTC connections are susceptible to developing unintended moments at the connection when subjected to displacements and rotations due to lateral loading (Stehn and Johansson, 2002). Most contemporary bolted BTC connection details result in tensile stresses parallel to the grain and longitudinal shear stress on the beam when subjected to lateral displacements and rotations (Lam, et al., 2008). These stresses can result in brittle failure mechanisms that lack ductility and are unfavorable for moderate to high seismic regions. Other traditional BTC connections such as timber rivets have been also been studied to quantify lateral deformation and ductility properties (Stahl, Wolfe and Begel 2004). The alternatives are true pinned connections, which employ a solid steel pin joining a steel bracket that is attached to each member. While appropriate for bridge structures, these are expensive to fabricate and require construction tolerances that are often economically impractical for repetitive gravity framing system.

A majority of past research efforts has been devoted to developing best practices for BTC connections with adequate ductility any displacement capacity to be used in moment frame structures. In addition to dowel-type fasteners, moment connections have been developed using steel rods epoxied to glulam beams and columns (Buchanan and Fairweather 1993). Rocking, non-linear seismic performance has also been achieved for moment-resisting timber connections by combining epoxied steel bars (fuses) and longitudinal post-tensioning tendons (Palermo, et al. 2008). While such connections allow for ductility and rotation capacity, they are intended to be part of the lateral load resisting system and could be costly to implement widely on all beam to column connections of a building. As such, options are limited for mass timber gravity BTC connections that are specifically designed to accommodate significant lateral drift. Such connections need to allow for the beam to rotate relative to the column during lateral deformations as a means of preventing excessive moment development at the connection. This independent beam rotation can also and minimize the development of unintended tensile stresses without 
reduction in gravity load capacity. This paper outlines one such option that was intended to be practical and economical.

The BTC connection was developed as part of the Framework Project, a proposed high-rise mixed use mass timber building located in Portland, OR (Zimmerman and McDonnel 2017). The Framework Project design utilized post-tensioned rocking shear walls as its LFRS, and thus the gravity connections are required to accommodate large displacement capacity that has not been available in conventional BTC connections. The connection was experimentally evaluated at full scale with the objectives of quantifying the lateral behavior while concurrently subjecting the connection to the design-level gravity loads in order to evaluate the performance during and quantify residual deformations following the imposed lateral displacements.

\subsection{CONNECTION DESCRIPTION AND TESTING PROCEDURE}

The BTC connection consisted of two separate components intended to perform different tasks: (1) a simple gravity load carrying bracket, and (2) a mechanical gap closure mechanism. Details and associated terminology are illustrated in Figure 1. The beam rests on a simple stiffened steel bracket that is nailed and screwed to the face of the column. The steel bracket supports the beam and the associated gravity loads. The end of the beam is notched such that the bracket is fully surrounded by the beam in the final installation, providing a fire-protective layer and an architecturally hidden connection. Since this connection bracket is rotationally unrestrained and lacks a positive lateral connection between the beam and column, a separate gap closure mechanism is installed on the top of the beam. This mechanism is comprised of a small steel bracket mounted on the top of the beam, a steel tension rod, plate washer and disc springs, also known as Belleville washers. This assembly is intended to impose a passive, displacement-induced restorative force to close horizontal gaps between the beam and column after lateral displacements. The intended connection behavior sought minimal rotational stiffness under seismic induced displacements. The connection was not intended to be part of the lateral force resisting system (LFRS), but rather was designed to accommodate the lateral drifts associated with design-level 
earthquake response of the building with minimal damage. The orientation of the Belleville washers, tension rod and plate washer produce tension forces that are parallel to grain in the beam, and compression force perpendicular to grain in the column.

Three experiments were conducted with the gravity load and lateral displacement protocol to evaluate the connection's performance, hereafter called Tests 1,2, and 3. The experiment used full-scale timber members and connection details to investigate lateral displacement behavior from expected seismic excitation using a quasi-static displacement protocol. The test setup simulated half-length of one fullheight bay of a typical story as illustrated in Figure 2. Although the specimens and connections were nominally the same for each of the three tests, selected variations on connection components were implemented as summarized in Table 1. The variations were chosen based on observations made from the preceding test in an effort to further refine the connection performance. The first modification reflected in Test 2 included a glued Teflon bearing pad at the bottom of the beam rather than loosely placed, smooth connection rod rather than all-thread rod, and $1.5 \mathrm{~mm}$ (1/16 in.) gap between the CLT and the column. The last variation reflected in Test 3 included changing the size and number of disc spring in order to increase the overall stiffness and deformation capacity in the gap closure mechanism.

All beams and columns were glulam timber members, the beams conforming to 24F-V4 (Douglas FirLarch) and the columns conforming to L2 (Douglas Fir-Larch) standards per American Wood Council National Design Specification 2015 Edition (NDS-2015). In each test, a full size $362 \mathrm{~mm}(14-1 / 4$ in.) x $419 \mathrm{~mm}$ (16-1/2 in.) x $3.52 \mathrm{~m}$ (11 ft. 6-3/4 in.) long column was connected to a full size beam spanning $4.27 \mathrm{~m}$ (14 ft.). with a full-sized $311 \mathrm{~mm}(12-1 / 4$ in.) x $610 \mathrm{~mm}$ (24 in.) cross section with an overall length of $4.27 \mathrm{~m}$ (14 ft.). This length corresponds to one-half of the $8.53 \mathrm{~m}(28 \mathrm{ft}$.) bay span of the proposed Framework Project building design.

Boundary conditions for all elements were chosen to isolate the structural behavior of the connection so as to be able to quantify the connection behavior. Schematic design of the test setup is summarized in Figure 3. The base of the column was connected using a pin, which is consistent with nominal intent of 
the detailing of the column splices in the proposed Framework building. Gravity load in the beam was simulated using hydraulic rams attached to tension rods, which were in turn anchored into the laboratory strong floor as shown in Figure 2. A slot was cut in the CLT panel to allow the rams to be placed directly on the top of the beam to ensure that all load applied by the rams was transferred into the beam and thereby the connection. This created a direct gravity load path between the rams, beam, and connection and mitigated potential for load to bypass the connection and transfer to the column via the CLT panel. The load was continuously monitored and adjusted to ensure that the applied gravity load in the connection remained at a constant demand of approximately $106.8 \mathrm{kN}$ (24 kips) irrespective of the lateral drift.

Under lateral drift, the beam rotates relative to the column. The resulting movement causes gaps to open as large rotations force the column and beam out of square. When displaced in one direction, a gap opens between the top of the beam and the column, compressing the disc springs and thereby engaging the gap closure mechanism. The compression stroke of the disc springs was designed to approximately the size of the gap anticipated at the design story drift. The compressed disc springs develop a clamping force between the beam and the column that opposes further rotation and impose a force to close the gap. The intent was to bring the beam and column back to the initial, plumb condition and prevent the beam from becoming unseated from the connection bracket. When displaced in the other direction, a gap opens between the bottom of the beam and the column and the gap closure mechanism is not engaged.

\subsection{IMPOSED LOAD AND DEFORMATIONS}

The lateral displacements were imposed using an actuator attached to the end of the beam opposite of the connection and in line with the seam between the CLT panel and the beam itself. Cyclic displacements followed a modified version of the CUREE W-02 (Krawinkler, et al., 2000) abbreviated displacement history. The target displacement, $\Delta$, was set to $3 \%$ drift, which was the expected inter-story drift under the Maximum Considered Earthquake $\left(\mathrm{MCE}_{\mathrm{R}}\right)$ for the proposed Framework Project. The Design Basis Earthquake (DBE) inter-story drift was $2 \%$, which also corresponds to a common code based drift limit 
(ASCE/SEI 7-10). The CUREE W-02 abbreviated displacement protocol was modified such that each primary cycle was followed by two trailing cycles of the same amplitude as the primary cycle. This deviation was made to investigate the effects of repeated deformation on the gap closure mechanism. The modified displacement protocol used for these experiments is summarized in Figure 4. Furthermore, additional drift cycles of $3 \%$ and $1.2 \%$ were added after the $4.5 \%$ story drift cycle to study the gap closure mechanism given that the disc springs were designed for only up to $3.0 \%$ drift. Each test concluded with three final cycles at $6 \%$ drift.

Instrumentation was installed on each specimen to monitor relative displacements between the beam and column, global lateral drift of the system, and global rotation of the column. The length of gaps opening between the beam and column were also measured during cyclic lateral displacements using LVDTs. Load cells measured the simulated gravity load applied to the beam, the vertical reaction in the far-end beam support, the applied lateral load, and the tension force developed in the gap closure mechanism.

Global coordinates for the test setup are assigned such that positive drift is considered as displacement in the positive longitudinal direction and negative drift refers to story displacement in the negative longitudinal direction as illustrated in Figure 3. Hence, positive drift cycles opened a gap at the top of the BTC connection and compressed the disc springs in the gap-closure mechanism, while negative drift cycles produced a gap at the bottom of the beam and did not engage the gap-closure mechanism. Rotation measurements follow standard counterclockwise sign convention. Actuator loads are shown as negative when in compression and positive when in tension. Connection shear in the direction of gravity is considered positive. The test setup was braced out-of-plane, constraining deformations to the plane of the setup.

\subsection{CYCLIC BEHAVIOR}

During each test, the lateral drift was measured and monitored in real-time to follow the prescribed displacement-controlled protocol. This real-time monitoring ensured that each cycle reached the target 
displacement regardless of any slack or slipping that may have occurred within the specimen. Rigid body movement was assumed and overall story displacement was calculated as $\delta_{\mathrm{s}}=\delta_{\mathrm{m}} *\left(\mathrm{H}_{\mathrm{s}} / \mathrm{H}\right)$, where $\delta_{\mathrm{s}}$ is the displacement at the top of the specimen $\delta_{\mathrm{m}}$ is the measured displacement at the height of the string pot displacement transducer, $\mathrm{H}$ is the height of the string pot above the center of rotation, and $\mathrm{H}_{\mathrm{s}}$ is the overall specimen height. Story drift, $\Delta_{\mathrm{s}}$, is expressed as a percentage of the overall story height, $\Delta_{\mathrm{s}}=$ $\left(\delta_{\mathrm{s}} / \mathrm{H}_{\mathrm{s}}\right) * 100 \%$.

The beam to column rotation was independently monitored in two different methods. The angle of rotation between the beam and the face of the column, $\theta$, was measured using a pair of LVDTs mounted directly to the beam. The angle of rotation at the base of the column, $\alpha$, was also measured using a pair of LVDTs. Figure 3 illustrates each of these rotation angles during a positive displacement cycle. Both measurement methods calculated rotations using trigonometric relationships and resulted similar rotation values for each test. The similarity in rotations calculated via each method indicated negligible deformation of the column and provided independent rotation measurements.

Shear force in the BTC connection was indirectly measured and recorded using the principles of engineering mechanics. The imposed gravity load on the beam along with the measured reaction at one end of the beam were used to calculate the shear in the gravity connection as $V_{c}=F_{a}+F_{r}+\left(W_{s} * L\right)-R$, where $V_{c}$ is the connection shear, $R$ is the measured reaction at the far end of the beam, $F_{a}$ is the selfweight of supporting the actuator, $\mathrm{F}_{\mathrm{r}}$ is the applied load from the hydraulic rams, and $\mathrm{W}_{\mathrm{s}}$ is the self-weight of the beam and floor slab. Connection shear was maintained at a constant level throughout the experiment to simulate the gravity loads carried regardless of lateral drift values achieved.

\subsection{Visual Observations}

During positive displacement cycles, a gap opened between the top of the beam and the face of the column as expected. This top gap is illustrated in Figure 5a and coincided with compression of the disc springs in the gap closure mechanism and the development of tension forces in the connection rod.

During and after drift cycles exceeding $4.5 \%$, the disc springs were observed to be squashed (did not 
return to their original size following compression) and no longer engaged the gap closure mechanism when the system returned to zero displacement. Negative displacement cycles produced a similar gap at the bottom of the beam. However, gaps caused by negative displacements were not intended and did not engage the gap closure mechanism located at the top of the beam.

No significant damage was observed in any of the experiments. Damage to the glulam members was superficial and localized to minor indentations in the surface of the column. A typical example of these minor indentations can be seen in Figure 5b following 6\% drift cycles, where the beam has been removed to expose the surface. The minor indentations occurred where the beam was bearing against the column face during positive displacement cycles. In all cases, the indents were measured to approximately 1-2mm deep and had negligible influence on the structure's overall performance. No cracks, no nail pullout or any other visible damage was observed to any of the structural members as a result of the experiments.

\subsection{Cyclic Force Deformation Behavior}

The recorded cyclic behavior for all tests are shown in Figure 6. Under repeated cyclic displacements, the force-displacement exhibits asymmetrical behavior of the BTC connection in tension relative to the compression. This is caused by the asymmetric nature of the BCT connection's gap closure mechanism. Free body diagrams of the beam and column at zero, at positive and at negative displacements are illustrated in Figure 7. Since the connection bracket is mounted to the side of the column, the gravity load reaction, $\mathrm{V}$, is always eccentric to the column's centerline. At zero displacement, this eccentricity produces a moment, $\mathrm{M}_{\mathrm{g}}$, which results in equal and opposite horizontal reactions at the base of the column and at the far side of the beam. The net result of this eccentricity is that for any gravity load, the beam is always in compression at zero displacement. This effect is evidenced by the negative (compressive) horizontal actuator loads shown for all tests in Figure 6 at zero drift.

For positive displacement cycles, $\mathrm{M}_{\mathrm{g}}$ continues to be present, but an additional moment, $\mathrm{M}_{\mathrm{c}}$, is introduced into the system as a result of the couple within the connection between the disc spring force, $\mathrm{T}_{\mathrm{s}}$, and the bottom of the beam bearing on the face of the column, $C_{b}$. During positive displacements, $M_{g}$ and $M_{c}$ 
have the same sign and are additive, meaning that as the disc spring force increases, so too does the compressive force in the beam. During the largest positive displacement cycle of each test (6\% story drift) the disc springs were fully compressed, which led to the rapid change in the connection's rotational stiffness evident as a dramatic spike in the moment-rotation plots for each test. In negative displacement cycles, $\mathrm{M}_{\mathrm{g}}$ and $\mathrm{M}_{\mathrm{c}}$ are also present, but $\mathrm{M}_{\mathrm{c}}$ develops from a couple with significantly different mechanical properties to the positive displacement cycles. Here, $\mathrm{M}_{\mathrm{c}}$ is the result of the top of the beam bearing on the face of the column, $\mathrm{C}_{\mathrm{t}}$, and the friction force developed between the beam and the connection bracket, $\mathrm{T}_{\mathrm{f}}$. During negative displacement cycles, $\mathrm{M}_{\mathrm{c}}$ and $\mathrm{M}_{\mathrm{g}}$ have opposite signs. As a result, $\mathrm{M}_{\mathrm{c}}$ reduces the compression in the beam caused by $\mathrm{M}_{\mathrm{g}}$. However, since the $\mathrm{T}_{\mathrm{f}}$ relies entirely on friction between the beam and BTC bracket, which itself is a function of the vertical gravity load, $\mathrm{M}_{\mathrm{c}}$ can never exceed $\mathrm{M}_{\mathrm{g}}$ while $\mathrm{V}$ remains in the direction of gravity. Furthermore, unlike the disc spring force, $\mathrm{T}_{\mathrm{s}}$, which develops as a function of the spring compression, $\mathrm{T}_{\mathrm{f}}$ is a friction force, and by definition, is constant as long as $\mathrm{V}$ remains constant. Thus, for negative displacement cycles, $\mathbf{M}_{\mathrm{c}}$ can reduce the compression in the beam, but only to the limit set by the maximum friction force, $\mathrm{T}_{\mathrm{f}}$. This is shown in the experimental data by the plateau in the force-displacement curves at negative displacements seen in each test. During these cycles, $\mathrm{M}_{\mathrm{c}}$ reduced the compressive force in the beam to the point where the beam began to slide on the bracket. Once this sliding began, the force-displacement shows that axial load in the beam remained relatively constant as evidenced by the plateaued portion of the curve in negative displacement cycles. This indicates friction is the only horizontal force acting in the connection during negative displacement cycles.

From this analysis, energy dissipation for positive displacements was shown to be confined to the compression of the disc springs while energy dissipation in negative displacement cycles was shown to be caused by friction losses between the beam and the bracket during sliding. These relationships are reflected in the hysteretic data and illustrate that the connection can dissipate energy during lateral displacement without causing flexural deformations to the gravity load carrying members. The critical 
gravity load path was shown to be capable of performing under large lateral displacements without degradation in gravity capacity. The lateral flexibility of the connection accommodated large displacements without significant damage to any structural members, demonstrating that the BTC connection is suitable for use in moderate to high seismic regions.

\subsection{MODELING OF GAP CLOSURE MECHANISM FORCE-DEFORMATION BEHAVIOR}

The gap closure mechanism generates forces within the BTC connection and can influence the overall behavior. The recorded force-displacement behavior for each test are shown in Figure 8, where the compression force generated by the disc springs is shown plotted against the lateral displacement of the connection rod relative to the top of the beam. These data reveal that at low positive displacement cycles (i.e. $1 \%$ drift and below) compression of the disc springs followed the repeatable, linear force-

displacement relationship shown at low displacements for all tests. As displacements were increased, the overall connection stiffness degraded and the disc springs began to show signs of plastic deformations, as evidenced by the stiffness reduction and permanent disc spring deformation after the connection was unloaded. With increase in displacements, the disc springs eventually fully compressed to the so-called flat load that was reached at approximately $4 \%$ drift for Tests 1 and 2 and 5\% drift for the Test 3 . The difference in drift levels between the tests is related to the disc spring size and quantities used in any of the particular tests. Once the flat load was reached, the force-displacement relationship was governed only by material properties of the glulam column and the steel connection rod. Accordingly, the connection's stiffness increased significantly above the flat load.

An analytical model was developed to quantify the distinct non-linear force-deformation relationship observed during connection tests. There are two different stiffness relationships considered by the model. The first contemplates a three-way series model that consists of the compressive stiffness of the disc springs, the compressive stiffness of the plate washer reacting on the glulam column, and the axial stiffness of the connection rod. Disc spring stiffness was calculated in accordance with DIN 2092, the 
European standard for determining force-deformation relationships in disc springs (DIN 2092, 2006).

The spring constant, $\mathrm{k}_{\mathrm{ds}}$, for an individual disc spring was calculated per Equation (1). All disc springs were stacked in series, thus the total disc spring stiffness for $n$ springs, $\mathrm{k}_{\mathrm{ds}}$, was computed per Equation (4). Here, $E_{d s}$ is the modulus of elasticity for the disc springs, $\mu$ is Poisson's ratio for the disc springs, $D_{e}$ is the disc spring outside diameter, $\mathrm{D}_{\mathrm{i}}$ is the disc spring inside diameter, $\mathrm{h}_{0}$ is the compressible height of a single disc spring, $\mathrm{t}$ is the thickness of the disc spring steel, $\mathrm{s}$ is the deflection of an individual disc spring and $\beta$ is the ratio of $D_{e} / D_{i}$.

$$
k_{d s}=\frac{4 E_{d s}}{1-\mu^{2}} * \frac{t^{3}}{K_{1} D_{e}^{2}} * K_{4}^{2} *\left(K_{4}^{2} *\left(\left(\frac{h_{0}}{t}\right)^{2}-3 * \frac{h_{0}}{t} * \frac{s}{t}+\frac{3}{2}\left(\frac{s}{t}\right)^{2}\right)+1\right)
$$

Where:

$$
\begin{gathered}
K_{1}=\frac{1}{\pi} * \frac{\left(\frac{\beta-1}{\beta}\right)^{2}}{\frac{\beta+1}{\beta-1}-\frac{2}{\ln (\beta)}} \\
K_{4}=1.0 \text { for springs with geometry used in this experiment } \\
K_{d s T}=\frac{1}{\left(\frac{n}{k_{d s}}\right)}
\end{gathered}
$$

The perpendicular-to-grain compressive stiffness of the circular plate washer on the DF L2 grade glulam column, $\mathrm{k}_{\mathrm{c}}$, could not be readily determined by codified engineering practice, so a representative value was determined experimentally. A piece of the timber glulam column that was used in the BTC was used to compress the same $76.2 \mathrm{~mm}$ diameter $\mathrm{x} 9.53 \mathrm{~mm}$ thick plate washer as used in the BTC tests. A total of 12 individual trials were conducted, all revealing an approximately bilinear force-displacement relationship typical for compression perpendicular to the grain. The elastic stiffness was determined for each of the trials using linear regression over a range of $0.25 \mathrm{~mm}$ to $1.0 \mathrm{~mm}$, which represented the relatively linear portion of the force deformation behavior. The average elastic compressive stiffness perpendicular to the grain under the circular washer from these tests was determined to be $26.3 \mathrm{kN} / \mathrm{mm}$. The axial stiffness of the connection rod, $\mathrm{k}_{\mathrm{r}}$, was determined using basic principles of engineering 
mechanics for an axially-loaded steel member. These three relationships were combined in series to analytically predict the force-deformation curve for the connection as a function of the Phase One stiffness, $\mathrm{k}_{1}$, as shown in Equation (5).

$$
k_{1}=\frac{1}{\frac{1}{k_{d s T}}+\frac{1}{k_{c}}+\frac{1}{k_{r}}}
$$

The second stiffness relationship considers the disc springs fully compressed and no longer contributing to the overall stiffness of the connection. This stroked out condition is described by stiffness, $\mathrm{k}_{2}$, per Equation (6).

$$
k_{2}=\frac{1}{\frac{1}{k_{c}}+\frac{1}{k_{r}}}
$$

The experimental data for Tests 1, 2 and 3 has been superimposed with the analytical model's prediction for each test in Figure 8, illustrating how the gap closure mechanism's force-displacement envelope in the connection can be mathematically predicted over the full experimental displacement range.

During each test, as positive displacement increased, plastic deformations began to develop in the disc springs. During the reverse cycles, this plastic deformation had reduced the overall height of the disc springs. Since the springs became shorter thorough this inelastic deformation, slack developed in the gap closure mechanism after larger displacement cycles. This inelastic deformation also revealed a reduced elastic stiffness of the disc springs on subsequent displacement cycles. The progressive stiffness degradation of the disc springs was attributed to the stress- and strain-gradients that develop within each individual spring because of its conical shape and is considered by the DIN 2019 stiffness model. Changing spring height, thickness and the number of springs used in the connection allowed the designers to select a combination for Test 3 that remained approximately elastic up to the design-level seismic drift of $2 \%$ (corresponding to a connection rod displacement of approximately $15 \mathrm{~mm}$ ). By contemplating the multi-step nonlinear stiffness of the gap closure mechanism, the analytical model was shown to accurately predict the gap closure mechanism force deformation behavior. The analytical model could be used to 
select the appropriate size and number of disc springs for future structures using this connection. Further, this model could allow future designers to accurately predict the forces that the BCT connection imposes on the beam and column during lateral displacement, which would aid in design of the gravity members and provide accurate envelope of connection forces for pushover analysis.

\subsection{EFFECTIVENESS OF GAP-CLOSURE MECHANISM}

In addition to accommodating large displacements with minimal rotational stiffness or damage, the disc springs were also included in the BTC connection as a means of providing a gap-closure mechanism between the beam and column. A restraint mechanism was required to address concerns that the beam could become unseated from the connection bracket during cyclical lateral displacements. Such a failure would cause significant damage to the CLT floor panels and pose danger of collapse. The gap-closure mechanism was designed to prevent such a failure. The disc springs were selected for their ability to impose a predictable force that would pull the end of the beam flush to the column face after lateral displacements. Such restorative behavior would mitigate the potential for large permanent displacements in the gravity system and help the building achieve overall seismic resiliency goals.

The aforementioned plastic deformations in the disc springs resulted in slack in the gap closure mechanism during negative cycles beyond $1 \%$ drift. The slack is visible in Figure 6 where permanent displacements increase with zero force in the connection for displacements larger than $1 \%$ drift. The number and thickness of disc springs present in the connection was shown to affect the magnitude of slack and the story drift at which slack began to develop. The slack within the disc springs resulted in a distinctive "S-shaped" unloading pattern in the connection's force-deformation curve during the transition between large negative (bottom gap) to positive (top gap) displacement cycles. This non-linear behavior occurred because the slack in the connection allowed a small gap at the top of the beam to open before the disc springs were compressed as sequentially illustrated in Figure 9. As lateral displacement increased, the disc springs developed a tension load that ultimately caused the beam to slide towards the column, producing the "S-shape" curve. 
Despite this s-shaped non-linearity, experimental results from each of the three tests showed minimal residual gap between beam and column even after cycles of $6 \%$ story drift. The residual gap between beam and column as measured at the connection bracket is shown in Figure 10. Here, each point on the curve represents the measured value of the gap in the BTC connection recorded in each drift cycle and includes both the gap at peak deformation and the residual gap, which occurs when the BTC connection returns to zero drift. The residual gap observed was negligible after displacement cycles within the elastic capacity of the disc springs (i.e. below $1.5 \%$ drift). However, at larger negative displacement cycles that created slack in the gap closure mechanism, residual gaps were observed between the beam and column. Figure 10 further illustrates the one-sided nature of the BTC connection. Positive displacement cycles were shown to always fully close gaps, even when slack had developed within the gap closure mechanism. This closure occurs because positive displacement cycles effectively push the beam and column together. The residual gaps that occur in negative displacement cycles above the linear threshold of the disc springs are linked to the magnitude of the displacement. These results imply that the residual gaps during negative displacement cycles are directly linked to the inelastic deformation of the disc springs. The designer could use the analytical model described above to select disc springs with an elastic limit appropriate to mitigate residual gaps at design-level displacements. Accordingly, the data in Figure 10 indicate that the BTC connection satisfies the target goals of mitigating permanent displacements after lateral loads preventing the beam from becoming unseated from the bracket due to compounding displacements.

\subsection{CONCLUSIONS}

The BTC connection was tested and found to be a practical and viable gravity connection for mass timber buildings with large lateral displacement demands. While not part of the LFRS, the connection was found to accommodate design-level seismic displacements and without reduction in gravity load carrying capacity and sustain minimal superficial damage. The connection's relative simplicity allow for easy constructability and utilization in structures that seek to meet similar displacement and load criteria. An 
analytical model was developed to predict the BTC connection's three-way series stiffness of the disc springs, column compression perpendicular to the grain, and axial tension of the connection rod.

Connection stiffness was shown to dramatically increase upon full compression of the disc springs as predicted by the analytical model. When adapting the BTC connection for use in other structures, the authors recommend that care is taken to ensure ample disc spring displacement capacity to avoid sharp changes in stiffness under seismic or wind loading. Variation of the number and size of disc springs in the gap closure mechanism was shown to affect the magnitude of gap in the connection and the drifts at which the gap began to develop. Results indicate that the gap caused by inelastic deformation of the disc springs could be minimized for design drifts through optimizing the number, height and thickness of disc springs used in the gap-closure mechanism. Gap closure was found to be largely a function of the connection's geometry and the eccentricity of the bracket to the centerline of the column. Additional testing may be required to fully evaluate if the gap-closure mechanism is required to prevent residual displacements between the beam and column.

\subsection{APPENDIX 1 - BEAM GROWTH EVALUATION}

Beam growth is an elongation of the distance between during lateral excitations columns that is a geometric result of rigid-body displacements and relative rotations between beams and columns. This compatibility issue is of particular consequence in simply supported frames where there is little rotational restraint between beams and columns. Beam growth can occur in this type of framed structure when a column rotates about its base in response to lateral loading or ground excitation. While the attached beam will translate with the column, due to its lack of rotational restraint, a gap will open between the face of the column and either the top or bottom the beam. This gap is additive to the physical length of the beam and in effect increases the distance between the supports on each side, causing the projected length of the beam between the columns to increase, defined henceforth as beam growth. In structural systems such as the BTC connection where the beam and column are displaced as rigid bodies with minimal member deformations, the size of the gap between these elements can be derived directly from the column rotation 
angle and the depth of the beam using trigonometry. Gravity connections in rocking structures must have adequate rotational deformation capacity and be capable of accommodating anticipated beam growth with negligible inelastic damage to the beams, columns, or connection elements. This requires additional detailing considerations that fall outside the scope of this text, but must be considered for structures that designed to meet rapid return to occupancy performance criteria.

The lack of rotational stiffness in the BTC connection means that both the beam and column exhibit negligible strains or deformations due to lateral displacements. This rigid body motion means that geometric relationships can be used to generate an analytical estimation beam grown based on measured connection rotation. The rotation of the connection, $\theta$, can be related to the gap at the top of the beam directly measured by a LVDT, $\mathrm{G}_{\mathrm{t}}$, and further extrapolated to overall beam growth, $\Delta_{\mathrm{b}}$ using basic trigonometry. The gap between beam and column at the level of either the top or bottom LVDTs, $\mathrm{G}_{\mathrm{t}}$, was predicted using geometric relationships and measured connection rotation angles by $\mathrm{G}_{t}=\mathrm{h}_{\mathrm{t}}[\tan (\theta)]$. Here, $h_{t}$ is taken as the distance from the bottom of the beam to the uppermost LVDT for positive displacement cycles and the distance from the top of the beam to the lowest LVDT for negative cycles. These predicted $\mathrm{G}_{\mathrm{t}}$ values were compared to the gaps directly measured using LVDTs for Tests 1,2 and 3. Results were found to be similar for each method, confirming that rigid body rotations between the beam and column could be assumed to accurately model and predict beam growth as a function of connection rotation or lateral displacement. Figure 11 shows the correlation between analytical and measured gaps using this procedure where positive gap values are at the top of the beam and negative gap values are at the bottom of the beam. This analytical method accurately predicted gap sizes at the locations of LVDTs on the test specimens. Thus, it was used to predict overall beam growth, $\Delta_{\mathrm{r}}$, by $\Delta \mathrm{r}=\mathrm{h}[\tan (\theta)]$, where $\mathrm{h}$ is the overall depth of the beam.

Using this method, beam growth at design-level displacements of $2 \%$ drift (corresponding to connection rotation of approximately 1.15 degrees) was predicted to be approximately $36.6 \mathrm{~mm}$. This illustrates that one limitation of the BTC connection is its susceptibility to beam growth. The BTC connection's 
minimal rotational stiffness allows for simple analytical predictions of beam growth as described above. This predictability means the engineer simply needs to know beam depth, the location of the connection bracket, and the anticipated rotations from DBE or MCE to accurately calculate beam growth within the BTC connection. The engineer can then determine how to best accommodate this growth to prevent unwanted connection damage. The authors propose further development of a connection that allows for horizontal displacements in excess of the predicted beam growth that could be used on the opposite side of the beam from the BCT connection to address beam growth-related concerns.

\subsection{APPENDIX 2 - FURTHER STUDY OF GAP CLOSURE MECHANISM}

In addition to Tests 1, 2, and 3 described above, three additional experiments (Tests 4, 5, and 6) were conducted to evaluate the performance of the gap closure mechanism with and without the applied gravity load. These additional tests used an abbreviated version of the displacement protocol from Tests 1, 2, and 3. Tests 4, 5, and 6 were not intended to validate the proposed BCT connection. These tests instead investigated the extent to which both the disc springs and eccentricity of the connection bracket mitigated the formation of residual gaps between the beam and column following cyclic displacement.

Test 4, 5, and 6 were all performed using the typical experimental setup and instrumentation described in the body of this report. All three tests used the same glulam beam, column and CLT panel that were used for Test 3 in the original BCT connection evaluation. In Test 4, new disc springs similar to those used in Test 3 were installed in the gap closure mechanism, however no gravity load was applied to the beam (other than self-weight). Test 4 followed an abbreviated pseudo-static cyclic deformation protocol up to $3 \%$ drift. The disc springs were removed for Test 5 , effectively eliminating the gap closure mechanism entirely from the connection. The simulated gravity load of $106.8 \mathrm{kN}$ was applied to the beam and the abbreviated displacement protocol was followed to 3\% drift. Finally, Test 6 eliminated both the disc springs and the applied gravity load and again followed the abbreviated displacement protocol to $3 \%$ drift. 
Each parametric test explored residual gap closure under a different connection condition to determine if the gap closure mechanism was actually required to prevent the formation of residual gaps. Peak and residual gaps were identified for each load cycle. These data are presented in Figure 12. The results illustrated here suggest that the gap closure mechanism is not explicitly responsible for preventing excess displacements in the connection. The residual gaps in the connection were negligible in size for Tests 46. The lack of significant residual gaps in Tests 4-6 reveals that the geometry of the connection as discussed in the Cyclic Force Deformation Behavior section of this paper plays a significant role in gap closures and enhances the effectiveness of the gap closure mechanism.

As previously discussed, the geometric and physical relationships between members in the BTC connection produce reactions that will always result in net compression between the beam and column. This has the effect of providing gap closure when the beam and column are pushed together at least in part as a result of the eccentricity of connection bracket. As previously discussed, Figure 7 summarizes the mechanics of this behavior under cyclic loading. Subsequently, one can expect limited residual displacements between the beam and column whether or not the gap closure mechanism is included in the connection. The disc springs instead were shown to be effective at reducing the connection's overall stiffness opposed to a more traditional bolted connection. This reduces moment transfer from the beam to the column and was experimentally shown to mitigate damage at story drifts well beyond code compliant levels. Further, the gap closure mechanism has been shown to be analytically predictable and thus allows the designer to refine member selection and more accurately model overall structural behavior during lateral displacements. The geometric advantage that is caused by the eccentricity of the beam load to the center line of the column only occurs in a one-sided application of BTC connection. It should be noted that the BCT connections tested in these experiments were all one-sided conditions. If the column were to have bays on either side of it, the eccentric loads would effectively balance and reduce the gap closure due to geometric advantages seen here. Thus, further research could explore the effectiveness of the gap 
closure mechanism in two-sided BTC connections where a clamping force may be required to prevent residual gaps and column unseating.

\subsection{DISCLAIMER}

Any opinions, findings, and conclusions or recommendations expressed in this material are those of the author(s) and do not necessarily reflect the views of the National Science Foundation.

\subsection{REFERENCES}

ANSI/AWC (American National Standards Institute/American Wood Council). (2015). "National Design Specification (NDS) for Wood Construction with 2015 Supplement.” NDS-2015, Leesburg, VA.

ASCE (American Society of Civil Engineers). (2010). "Minimum Design Loads for Buildings and Other Structures.” ASCE/SEI 7-10, Reston, VA.

Buchanan, A., Deam, B., Fragiacomo, M., Pampanin, S., \& Palermo, A. (2008). Multi-storey prestressed timber buildings in New Zealand. Structural Engineering International, 18(2), 166-173.

Buchanan, A. H., \& Fairweather, R. H. (1993). Seismic design of glulam structures. Bulletin of the New Zealand national society for earthquake engineering, 26(4), 415-436.

Chang, W. S., Hsu, M. F., \& Komatsu, K. (2006). Rotational performance of traditional Nuki joints with gap I: theory and verification. Journal of wood science, 52(1), 58-62.

German Institute for Standardization (Deutsches Institut für Normung). (2006). "Disc springs Calculation.” DIN 2092 (2006-03), Berlin.

Jorissen, A., \& Fragiacomo, M. (2011). General notes on ductility in timber structures. Engineering structures, 33(11), 2987-2997. 
Krawinkler, H., Parisi, F., Ibarra, L., Ayoub, A., \& Medina, R. (2000). Development of a testing protocol for wood frame structures, CUREE Publication No. W-02, California.

Lam, F., Schulte-Wrede, M., Yao, C. C., \& Gu, J. J. (2008). Moment resistance of bolted timber connections with perpendicular to grain reinforcements. Proc. 10th WCTE Miyazaki, Japan, CD-ROM Proceedings.

Stahl, D. C., Wolfe, R. W., \& Begel, M. (2004). Improved analysis of timber rivet connections. Journal of Structural Engineering, 130(8), 1272-1279.

Stehn, L., \& Johansson, H. (2002). Ductility aspects in nailed glue laminated timber connection design. Journal of Structural Engineering, 128(3), 382-389.

\subsection{FIGURES}

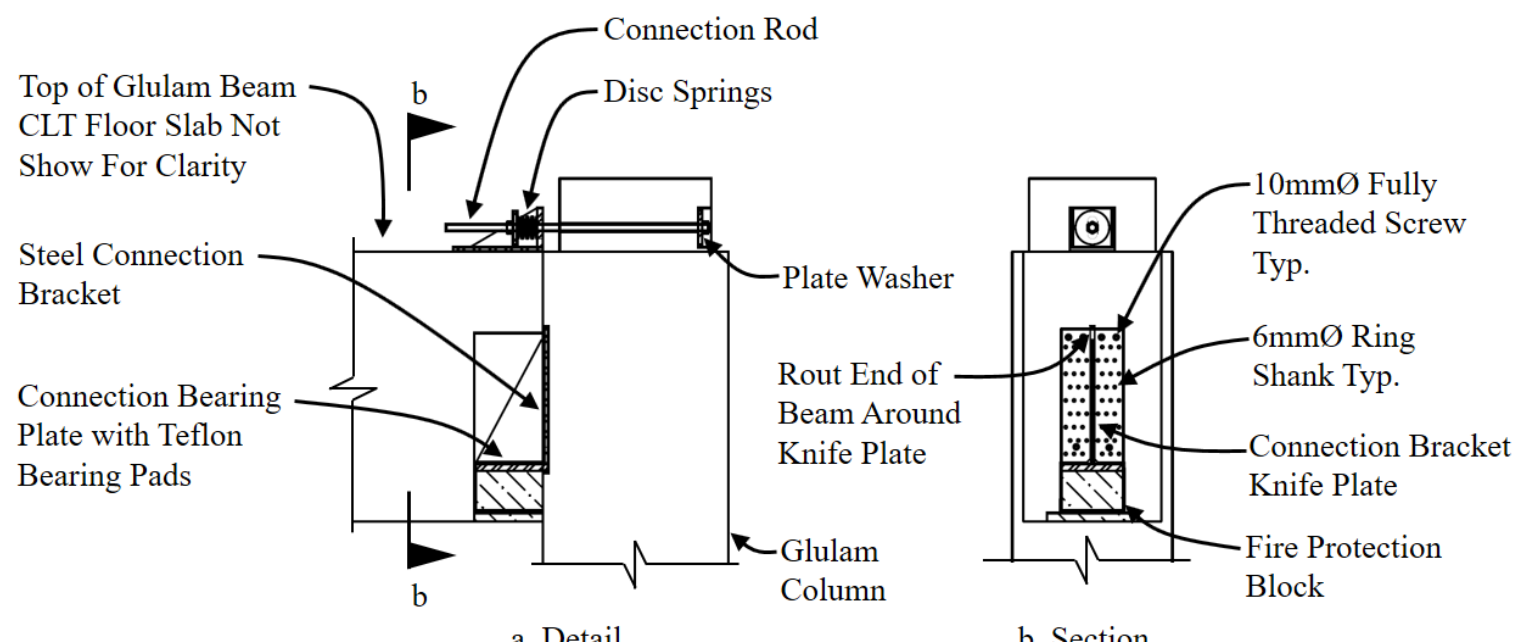

Figure 1 - Detail and section of BTC connection. 


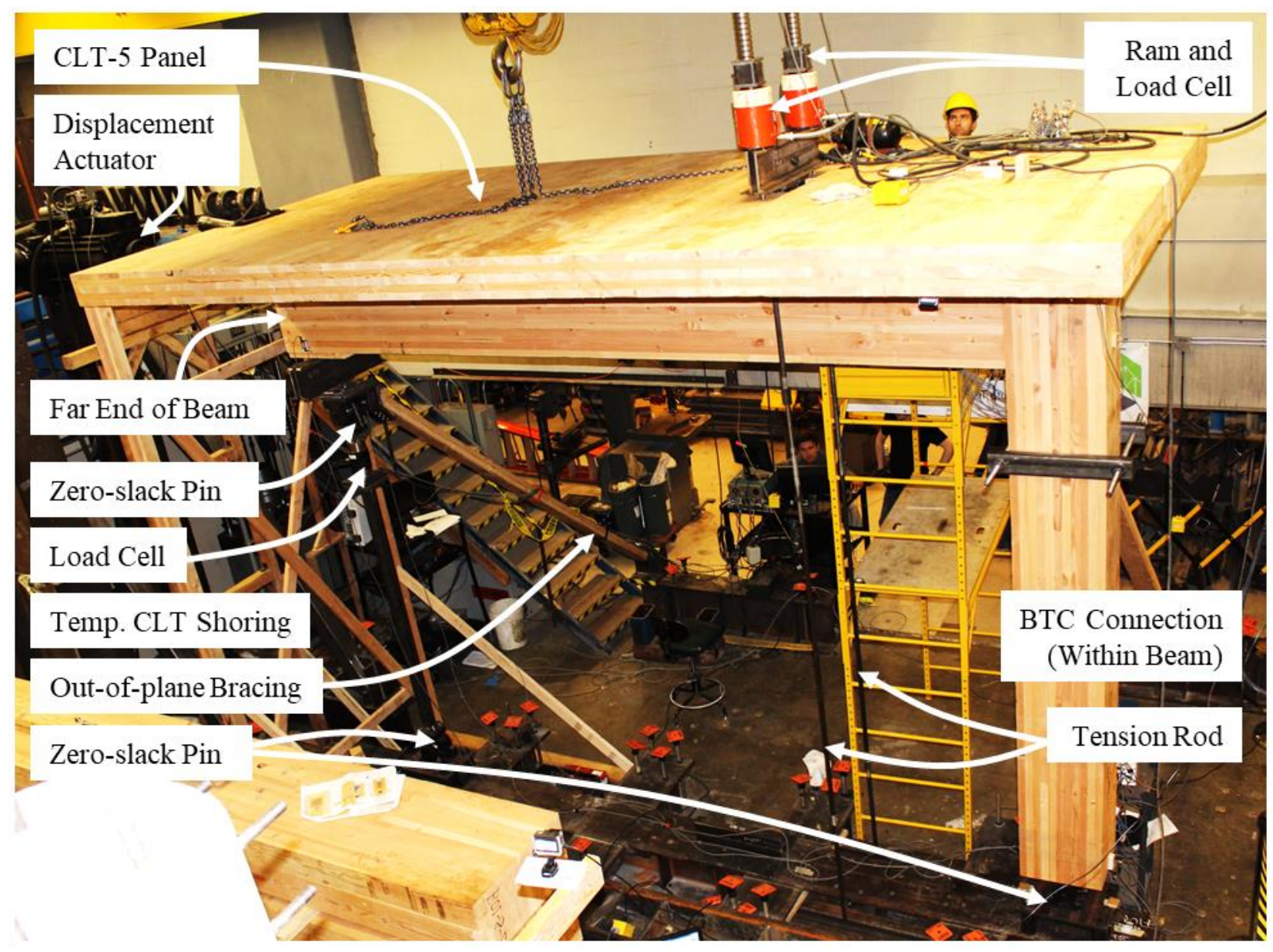

Figure 2 - Full-scale test setup in laboratory. 


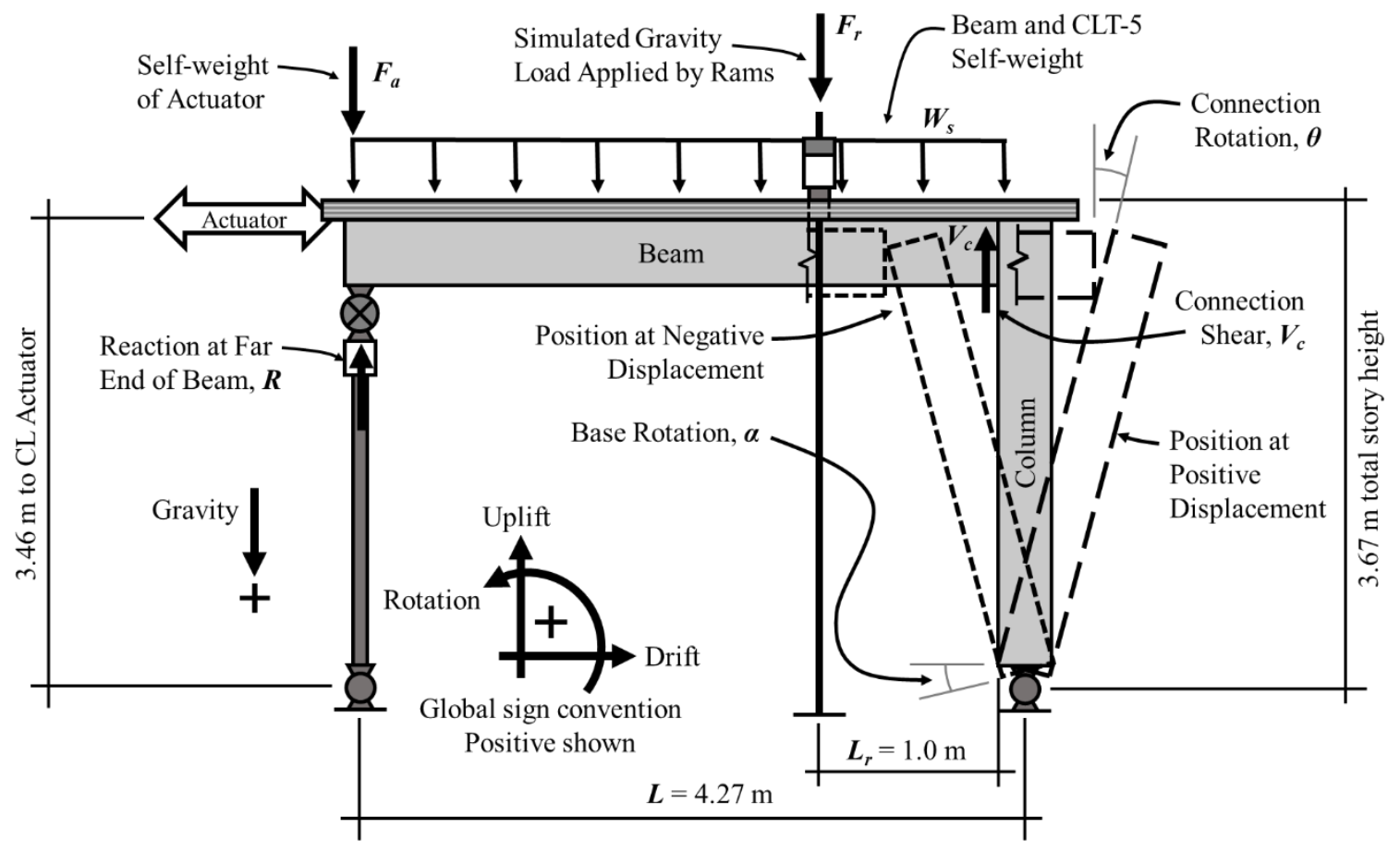

Figure 3 - Test setup overview and free body diagram.

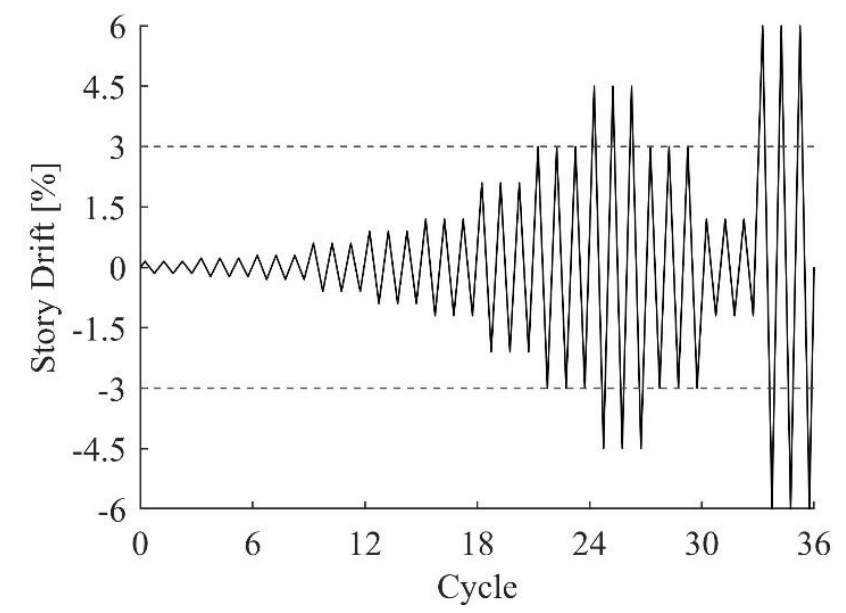

Figure 4 - Displacement history protocol modified from CUREE W-02. Expected drift from MCE shown as dashed line. 

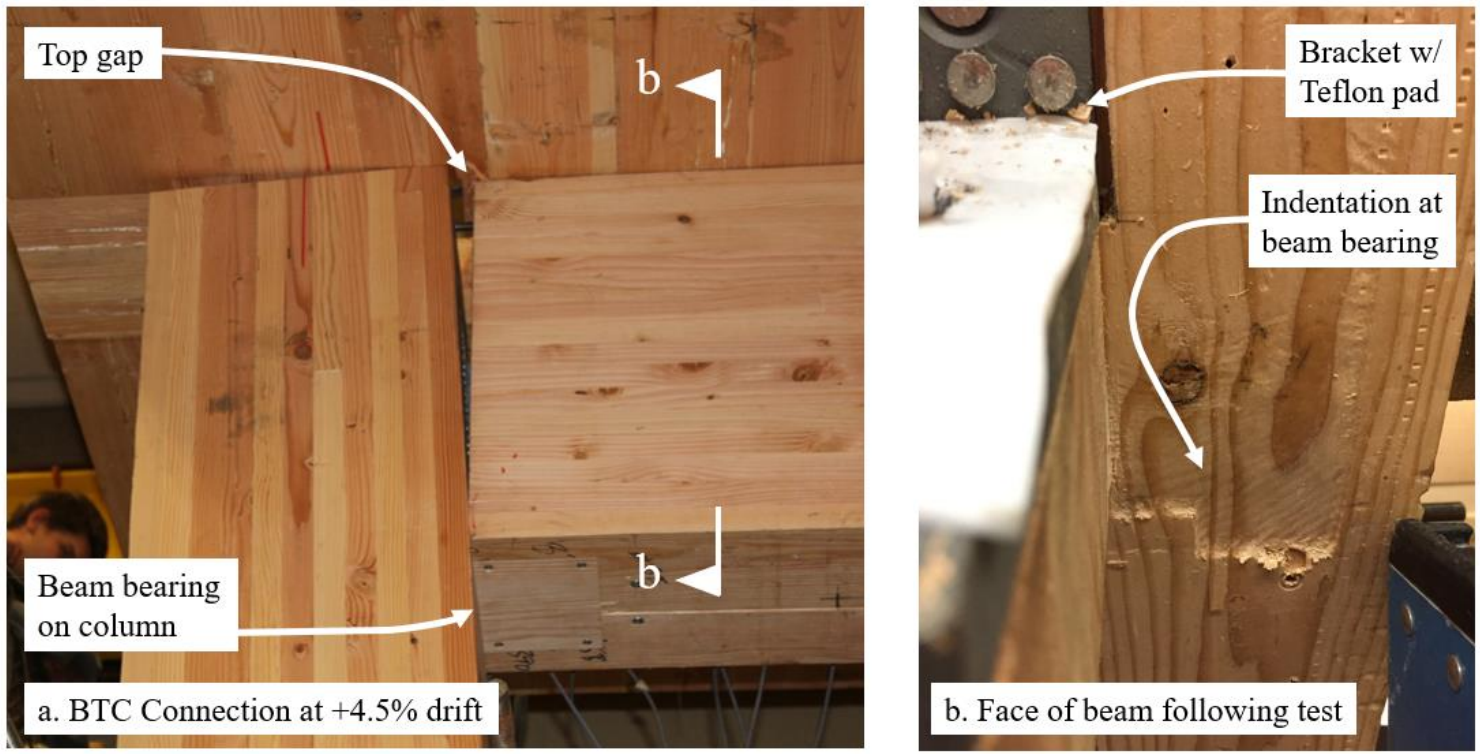

Figure 5 -BTC Connection During $6 \%$ Positive Drift and Visible Indentation in Column Face. 


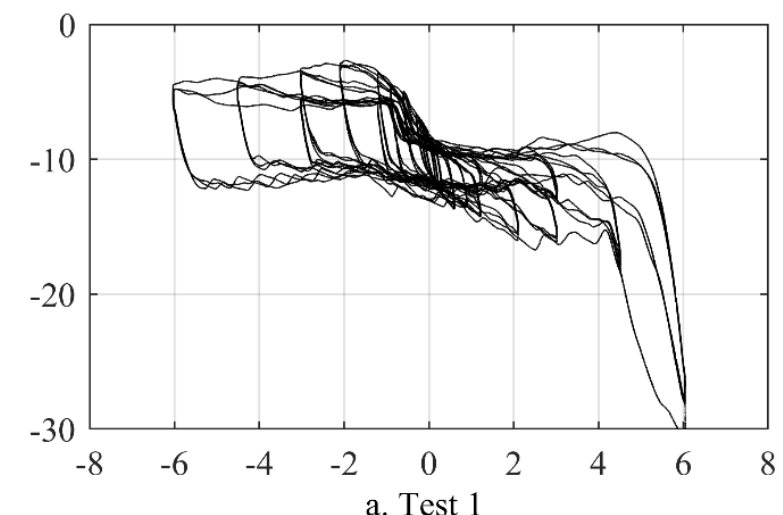

a. Test 1
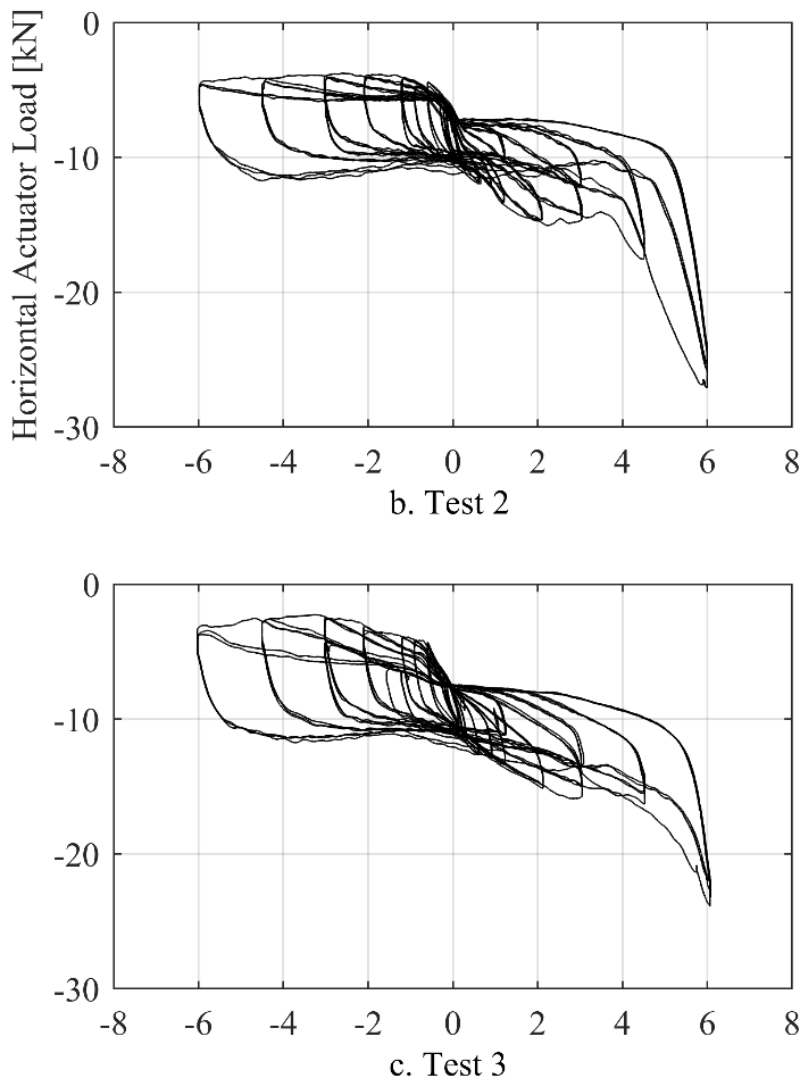

Story Drift [\%]

Figure 6 - Force vs. dirft plots for BTC connection tests. 

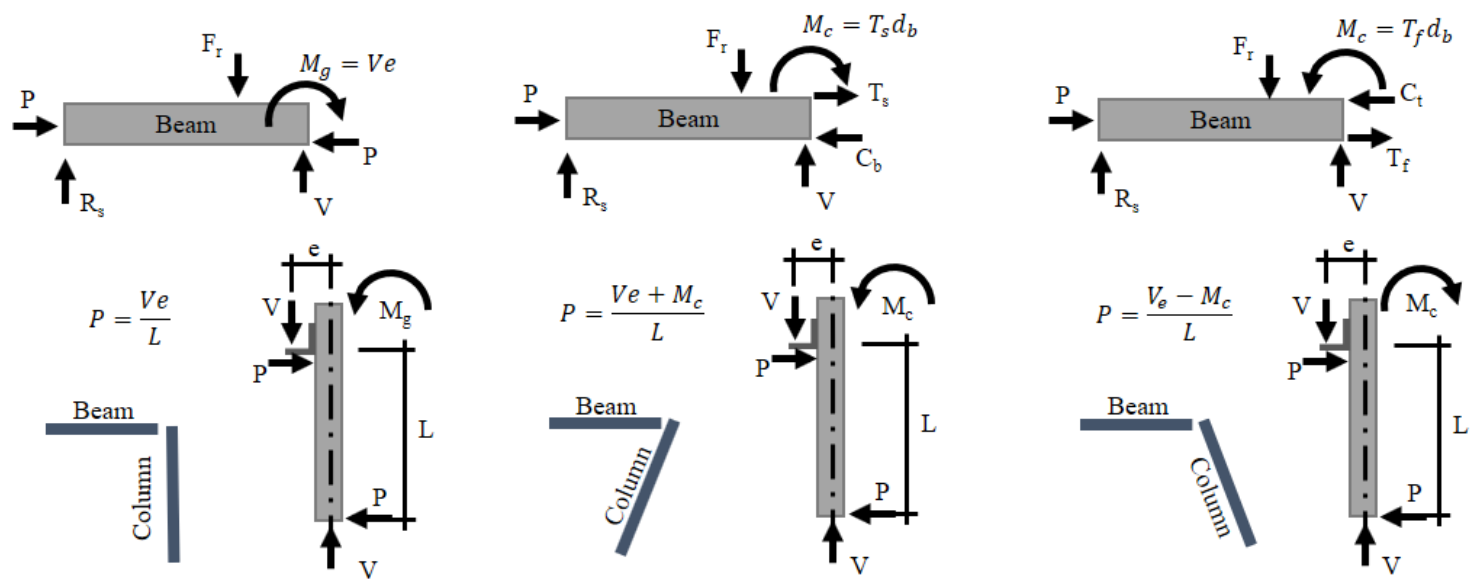

a. At Zero Displacement

b. At (+) Displacement

c. At (-) Displacement

Figure 7 - BTC Connection free body diagram. 


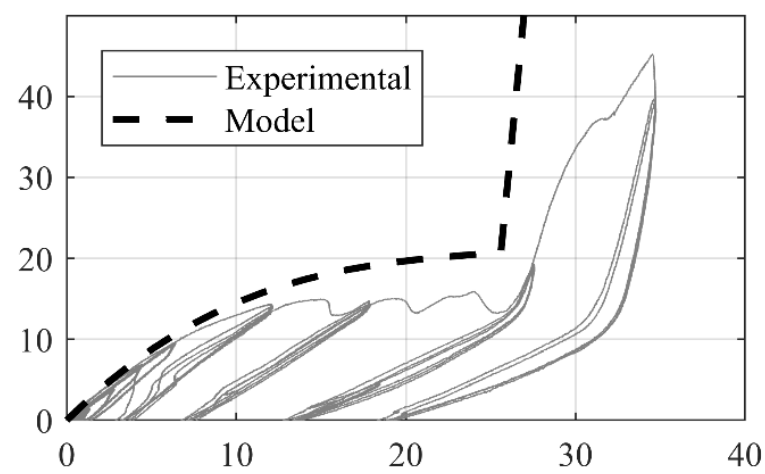

a. Test 1
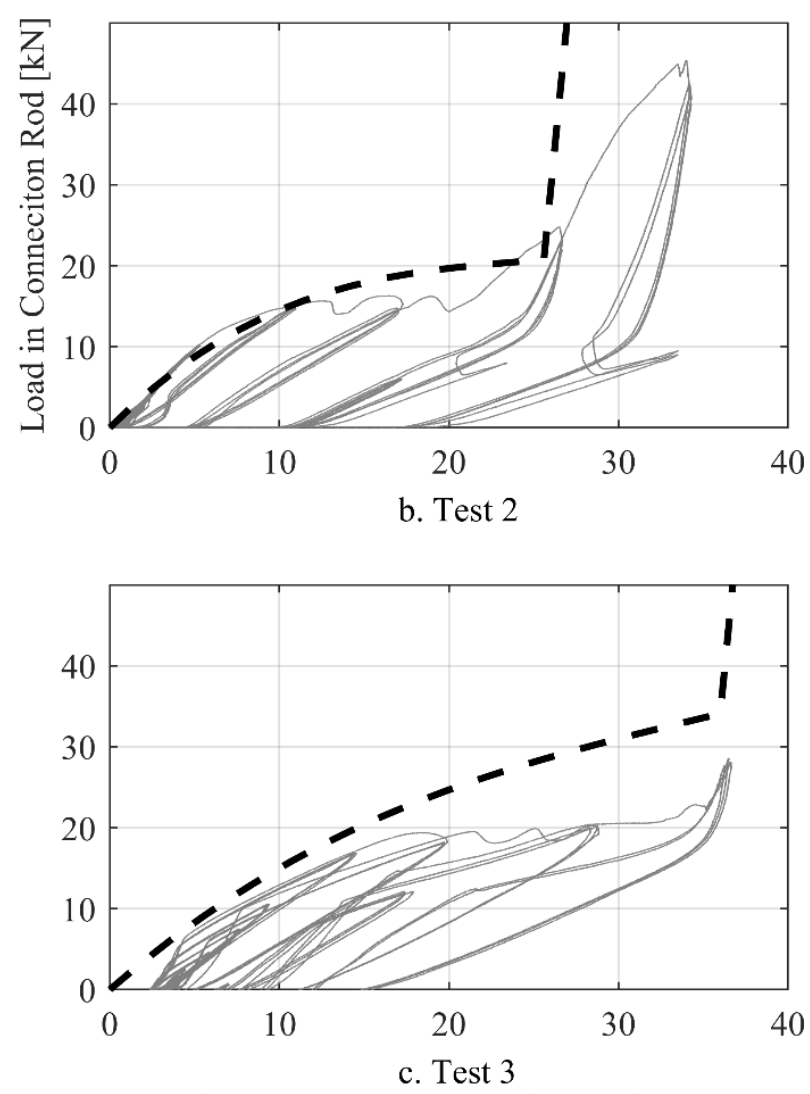

Displacement at Connection Rod [mm]

Figure 8 - Experimental and analytical connection force-displacement plots for BTC connection. 

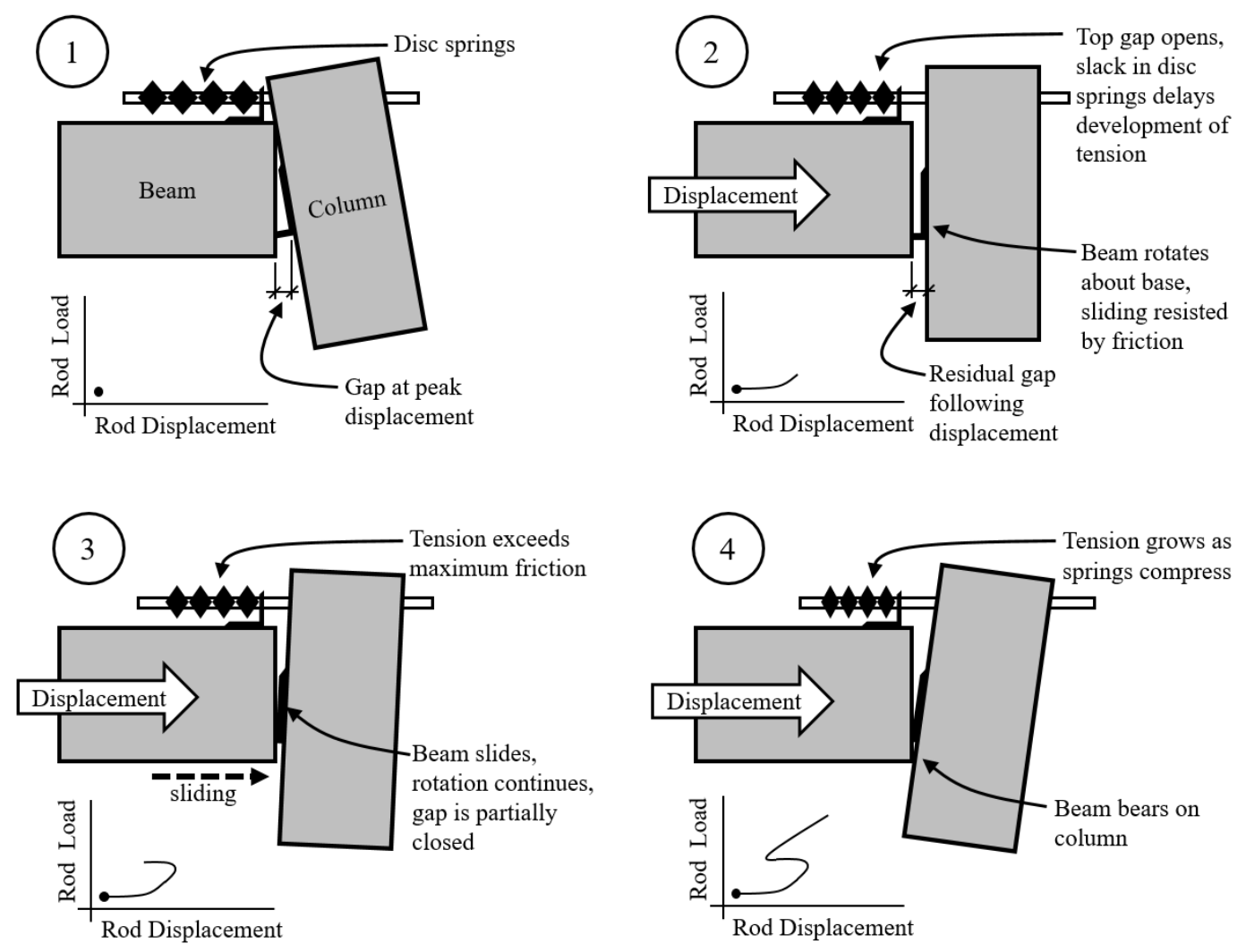

Figure 9 - Non-linear develpment of S-shaped force-displacement curve. 

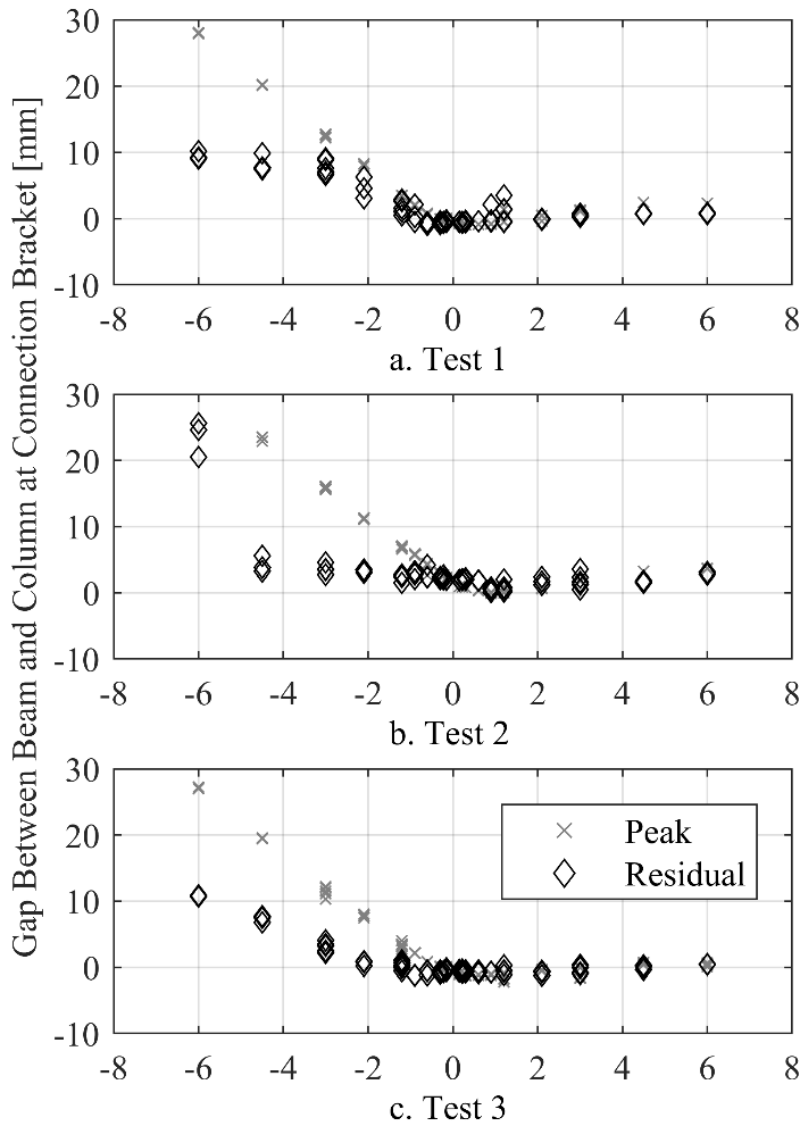

Story Drift Displacement Cycle [\%]

Figure 10 - Peak and residual gaps between beam and column. 

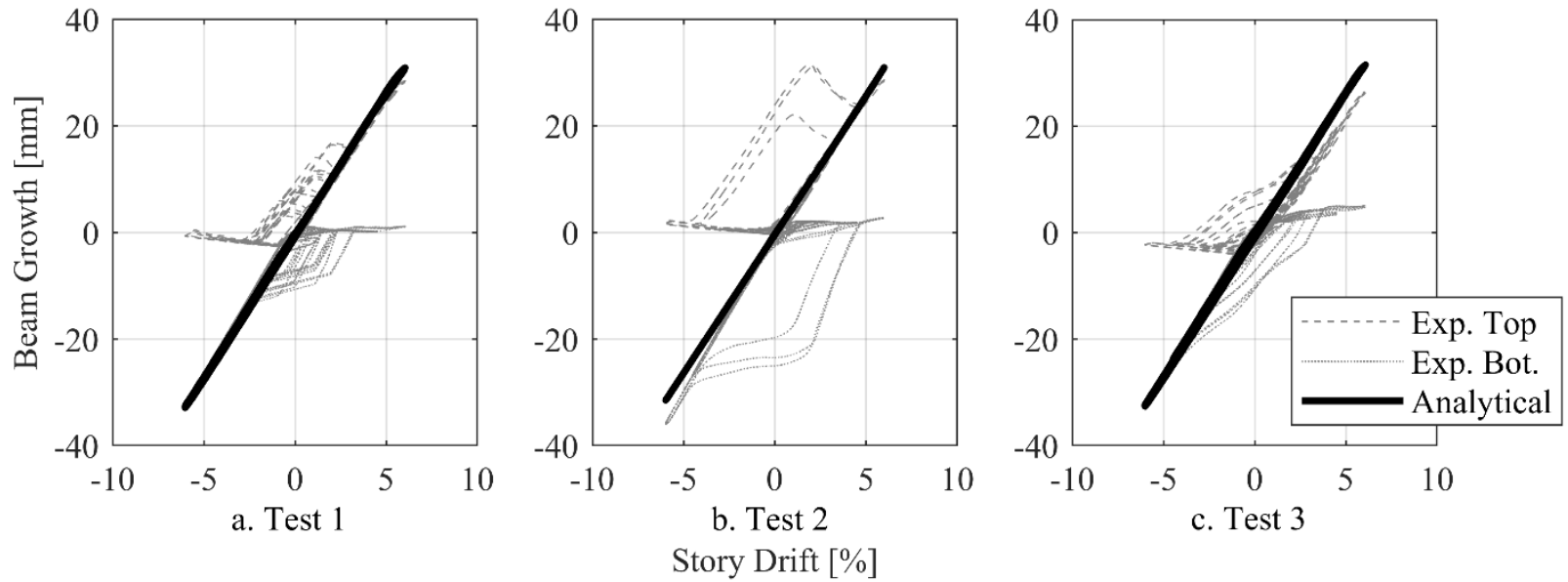

Figure 11 - Experimental and analytical beam growth evaluation.

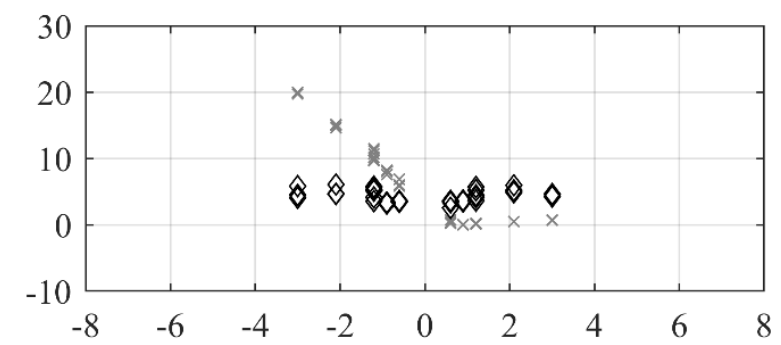

d. Test 4 - No Load, With Springs

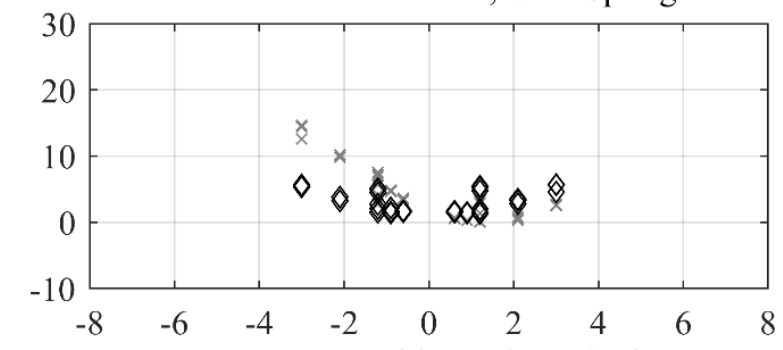

e. Test 5 - With Load, No Springs

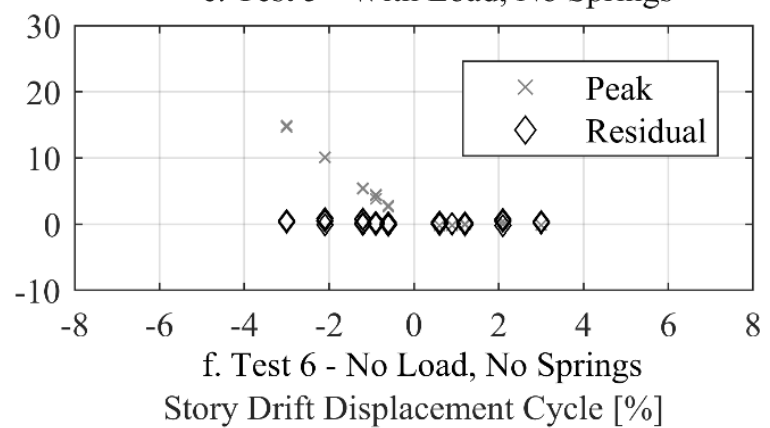

Figure 12 - Peak and residual gaps between beam and column for parametric tests. 


\subsection{TABLES}

Table 1 - Variable conditions for each BCT Connection test.

\begin{tabular}{|c|c|c|c|}
\hline Variable & Test 1 & Test 2 & Test 3 \\
\hline Specimens & B01 \& C01 & B02 \& C02 & B03 \& C03 \\
\hline Beam Moisture Content & $9.4 \%$ & $7.7 \%$ & $8.0 \%$ \\
\hline Col. Moisture Content & $8.8 \%$ & $7.7 \%$ & $7.8 \%$ \\
\hline Teflon Bearing Pads & Unglued & Glued & Glued \\
\hline Column to CLT Gap & None & $1 / 16 "$ & $1 / 16 "$ \\
\hline Number of Disc Springs & 8 & 8 & 12 \\
\hline Disc Spring Thickness & $2.5 \mathrm{~mm}$ & $2.5 \mathrm{~mm}$ & $3.0 \mathrm{~mm}$ \\
\hline Disc Spring Height & $5.7 \mathrm{~mm}$ & $5.7 \mathrm{~mm}$ & $6.0 \mathrm{~mm}$ \\
\hline Connection Rod & 5/8" all-thread & $\begin{array}{l}5 / 8 \text { " smooth rod with } \\
\text { long threaded ends }\end{array}$ & $\begin{array}{l}5 / 8 \text { " smooth rod with } \\
\text { short threaded ends }\end{array}$ \\
\hline
\end{tabular}

